Muséologies

Les cahiers d'études supérieures

muséologies

\title{
L'art autochtone à l'aune du discours critique dans les revues spécialisées en arts visuels au Canada. Les cas de Sakahàn et de Beat Nation
}

\section{Edith-Anne Pageot}

Volume 9, numéro 1, 2018

Monde des Arts / Arts des mondes

URI : https://id.erudit.org/iderudit/1052629ar

DOI : https://doi.org/10.7202/1052629ar

Aller au sommaire du numéro

Éditeur(s)

Association Québécoise de Promotion des Recherches Étudiantes en Muséologie (AQPREM)

ISSN

1718-5181 (imprimé)

1929-7815 (numérique)

Découvrir la revue

Citer cet article

Pageot, E.-A. (2018). L'art autochtone à l'aune du discours critique dans les revues spécialisées en arts visuels au Canada. Les cas de Sakahàn et de Beat Nation. Muséologies, 9(1), 81-94. https://doi.org/10.7202/1052629ar
Résumé de l'article

This article offers a qualitative and quantitive analysis of the critical reception of two exhibitions, Sakahàn:International Indigenous Art (National Gallery of Canada, Ottawa 2013) and Beat Nation: Art, Hip-Hop and Aboriginial Culture (organised and circulated by the Vancouver Art Gallery, 2013-2014). The study treats articles which appeared between 2012 and 2015 in English and French visual-arts publications. The comparative analysis intends to highlight general trends, in order to identify challenges that contemporary Indigenous arts pose for art criticism. A review of the texts shows that all commentators, whether francophone or anglophone, indigenous or non-Indigenous, have welcomed these two exhibitions warmly. The discrepancy between the number of essays in French and those in English reflects the demographic weight of these two linguistic communities and the geographic distribution of First Nations in Canada. This will qualify, without denying, the hypothesis of Quebec's tardiness on the indigenous question. The authors largely recognize the necessity of initiating indigenization of the museum and emphasize the movement to internationalize contemporary indigenous art. Yet many commentators, particulary Indigenous people, dispute the efficacity of the concept of "strategic essentialism" put forward by the commissioners of the Sakahàn catalog. Despite both a real interest in these two major exhibitions and the quality of the commentary, in the end, for events of such a scale few texts have been published on the subject. The criteria for appreciation rooted in the institutional sociology of art endeavour to fully take into account the challenges posed by certain central aspects of the approach of several Indigenous creators, such as the intangible dimensions of their civic engagement, the dissolution of particular outside venues and the sisterhood of certain projects.
Tous droits réservés ( Association Québécoise de Promotion des Recherches Étudiantes en Muséologie (AQPREM), 2018
Ce document est protégé par la loi sur le droit d'auteur. L’utilisation des services d'Érudit (y compris la reproduction) est assujettie à sa politique d'utilisation que vous pouvez consulter en ligne.

https://apropos.erudit.org/fr/usagers/politique-dutilisation/ 
Article quatre

L'art autochtone à l'aune du discours critique dans les revues spécialisées en arts visuels au Canada. Les cas de Sakahàn et de Beat Nation

Edith-Anne Pageot 
Édith-Anne Pageot est professeure au Département d'histoire de l'art de l'Université du Québec à Montréal, membre régulier de l'Institut de recherches et d'études féministes (IREF) et du Centre de recherche interuniversitaire sur la littérature et la culture québécoises du (CRILCQ). Elle est également professeure auxiliaire et membre de la Faculté des études supérieures et postdoctorales de l'Université d'Ottawa où elle fut lauréate du Prix d'excellence en enseignement en 2013. Spécialiste des modernités au Québec et au Canada, elle s'intéresse aux politiques de l'identité et à ses formes complexes. Elle s'interroge sur les manières dont les images et les dispositifs de médiation culturelle façonnent les concepts de genre, de territoire, de nation et de collectivité. Elle dirige actuellement le projet de recherche, La culture artistique au Collège Manitou : agentivité et stratégies d'autodétermination (CRSH 2017-2019). Parmi ses plus récentes publications, mentionnons: «Postcolonial Territorial Landmarks within Canada's Multiculturalism: The Myth of Virility». Wilfrid Laurier Press (2017) et «Figure de l'indiscipline. Domingo Cisneros, un parcours artistique atypique " RACAR Revue d'art canadienne/Canadian Art Review 42.1 (2017) 
Entre 2012 et 2014, deux expositions majeures d'arts autochtones contemporains ont été présentées dans différentes villes canadiennes. L'envergure internationale et l'importance historique de ces événements tenus dans de grands musées québécois et canadiens - dont deux sont des musées d'État - promettaient une réception critique féconde. L'exposition collective Sakahàn. L'art indigène international a été présentée au Musée des beaux-arts du Canada (Ottawa) du 17 mai au 2 septembre 2013. Décrite comme la plus grande exposition d'art autochtone contemporain jamais réalisée par le Musée, la première d'une série quinquennale, elle réunissait quelque 150 œuvres, 80 artistes provenant de 16 pays. Elle a été élaborée à partir d'un travail de collaboration entre trois commissaires, Greg Hill (Kanyen'kehaka, membre du Territoire des Six Nations de la Rivière Grand, conservateur de l'art autochtone, MBAC), Candice Hopkins (membre de la Première Nation de Carcross-Tagish, conservatrice invitée) et Christine Lalonde (conservatrice associée, estampes et dessins canadiens, MBAC), avec l'appui de plusieurs commissaires-conseils d'origine autochtone, provenant de différents pays. La version muséale de Beat Nation art, hip-hop et culture autochtone a été, quant à elle, organisée et mise en circulation dans six villes canadiennes, entre 2012 et 2014, par la Vancouver Art Gallery, à partir d'une initiative de la Grunt Gallery, un centre d'artistes fondé en 1984 à Vancouver. Elle a été conçue par les commissaires Kathleen Ritter (artiste et commissaire associée à la Vancouver art Gallery de 2007 à 2012) et Tania Willard (artiste, membre de la Première Nation Secwepemc). Elle réunissait 27 artistes provenant du Canada et des États-Unis, de l'Alaska en passant par le Nunavut, l'est du Labrador et, au sud, par le Nouveau-Mexique.
Étant donné l'ampleur de ces expositions et leur caractère exceptionnel dans le paysage canadien, on pouvait s'attendre à ce qu'elles suscitent une réflexion approfondie sur les défis que pose l'art autochtone contemporain à la critique d'art et au musée. À ce sujet, rappelons qu'à compter de 1992 déjà, le musée est identifié comme l'un des principaux espaces à partir desquels on a mesuré la logique et les structures de discrimination, d'exclusion et l'impérialisme culturel du régime colonial. En effet, à l'issue de son examen de la situation canadienne, le Groupe de travail sur les musées et les Premières Nations constate, en 1992, le manque d'implication des Autochtones dans l'interprétation de leurs cultures et de leurs histoires, leur sous-représentation criante dans toutes les sphères d'activité des musées, allant de la demande de subvention à la planification des expositions, aux mécanismes d'acquisition jusqu'aux plus hautes instances ${ }^{1}$. Devant ces états de la situation, on en appelle à une réforme profonde. Selon les recommandations formulées dans son rapport, le Groupe de travail considère qu'il faut " tourner la page " (c'était le titre du rapport), faire du musée un espace susceptible d'enclencher des changements et mettre en œuvre de nouveaux cadres éthiques et de travail qui favorisent l'intégration sociale et la responsabilité ${ }^{2}$. Les conclusions récentes de la Commission sur la vérité et la réconciliation au Canada (2010-2015) abondent dans le même sens: " Grâce à leurs expositions, leurs activités de sensibilisation et leurs programmes de recherche, tous les musées occupent une place privilégiée pour contribuer à une éducation en vue de la réconciliation ${ }^{3}$ ". Aujourd'hui, une nouvelle génération d'artistes-commissaires d'origine autochtone est directement impliquée dans la coordination d'événements artistiques nationaux et internationaux. La nomination en 2007 du commissaire et artiste d'origine mohawk Greg A. Hill à la tête de la Chaire de

2 À ce sujet, voir notamment: MESSAGE Kelly, New Museums and the Making of Culture. Oxford: Berg, 2006. 3 Rapport final de la Commission sur la vérité et la réconciliation du Canada, 2015, p. 280. http://nctr.ca/assets/reports/ Final\%20Reports/French_Executive_Summary_Web.pdf (consulté le 2 mai 2016).
1 ERASMUS Georges, FLEWWELLING Morris F., MERCREDI Ovide, HILL Tom, " Tourner la page: Forger de nouveaux partenariats entre les musées et les Premières nations. Rapport du Groupe de travail sur les musées et les Premières Nations ". Ottawa: Assemblée des Premières Nations et Association des Musées canadiens, (1992) 1994 (3e éd.). 
conservation de l'art indigène Audain demeure un exemple probant ${ }^{4}$. Ces voix espèrent contribuer à redéfinir les pratiques de conservation, de collection et d'expositions, afin d'ébranler les fondements colonialistes qui précèdent à la mise en valeur et à la contextualisation des œuvres produites par des créateurs d'origine autochtone. Majoritairement conçues par des commissaires autochtones, Sakahàn et Beat Nation ont été en quelque sorte des porte-paroles de ces voix et constituent des tentatives de repenser la présence autochtone dans le musée. Qu'en est-il de la critique d'art?

Depuis plusieurs décennies déjà, la déconstruction des mythes modernistes a réaffirmé la dépendance de l'objet d'art sur ses conditions d'exposition, incluant l'activité critique. Lié à l'exposition, le commentaire critique revêt un potentiel performatif et constructiviste que plusieurs chantiers de recherche ont réexaminé. Les sociologues de la médiation ont montré que l'art n'est pas qu'une expérience singulière, mais aussi une expérience collective façonnée par des contraintes matérielles et sociales au sein de laquelle la question de la reconnaissance est centrale ${ }^{5}$. Dans son livre aujourd'hui notoire, qui a d'ailleurs inspiré le sujet du colloque et qui a donné naissance à ce numéro thématique, Howard S. Becker insiste sur le fait que la réalisation d'une œuvre «ne se conçoit pas sans la présence d'un public qui réagit et porte une appréciation $^{6}$ ». Dans son avant-propos du livre, La critique d'art en France, 1850-1900, Jean-Paul Bouillon rappelle pour sa part le fait que, «[...] l'œuvre d'art ne commence vraiment à vivre de la vie sociale à laquelle elle $\mathrm{a}$ vocation qu'à partir du moment où elle est vue, donc commentée: la critique n'est jamais qu'un simple écho, plus ou moins déformant, elle est,

4 La création de la chaire Audain, soutenue financièrement par le secteur privé, crée un précédent dans le milieu muséal public au Canada. Un jour, il faudra réfléchir aux enjeux, aux possibilités et aux contraintes qu'offre ce modèle de financement.

5 BAL Mieke. "Guest Column: Exhibition Practice ". PMLA, vol. 125, $\mathrm{n}^{\circ}$ 1, 2010, p. 9-23.

6 BECKER Howard, S. Les mondes de l'art. Paris: Flammarion, 1988, p. 30. Ce livre est d'abord paru en anglais, Art Worlds, en 1982 aux éditions University of California Press. parmi d'autres, un élément constitutif de l'objet lui-même ${ }^{7}$ ". Pour Nathalie Heinich, les commentaires sur l'art contribuent à « la montée en objectivité ${ }^{8}$ ", autrement dit ils constituent des "mécanismes de valorisation " qui font de certaines catégories d'objets ou de gestes, des œuvres d'art reconnues comme telles par autrui. Plus récemment, Gilles Lipovetsky insiste, quant à lui, sur le fait que dans le régime mondial actuel, aujourd'hui plus que jamais, les opérations de communication et de médiation culturelle, dont la critique d'art est partie intégrante, participent aux conditions sociales d'existence de l'objet ${ }^{9}$. Bref, si l'activité critique est l'un des facteurs constitutifs de l'art contemporain, on peut se demander quelle place a réservée la presse canadienne spécialisée en arts visuels à Sakahàn et à Beat Nation. Quels sont les principaux enjeux soulevés par les auteurs qui ont écrit sur le sujet? Quels sont leurs principaux angles morts?

Pour répondre à ces questions, nous avons procédé à la recension des articles portant sur ces deux expositions importantes et parus dans les revues spécialisées en arts visuels, avec ou sans arbitrage par les pairs, en version numérique et imprimée. Ces diffuseurs sont d'importants acteurs d'homologation de l'art contemporain. Pour cette raison, il nous semblait pertinent d'entamer l'examen du discours critique en commençant par ces réseaux. De plus, les textes qui y sont publiés, généralement plus longs que les comptes rendus descriptifs diffusés par les quotidiens, nous paraissaient propices à l'élaboration par les auteurs d'une réflexion un peu plus approfondie qui aurait pu révéler l'intérêt de la critique d'art à envisager, à discuter et à interroger les mécanismes visant le processus d'indigénisation du milieu de l'art au Canada.

7 BOUILLON Jean-Paul. La critique d'art en France 18501900. Saint-Étienne: Université de Saint-Étienne, 1989, p. 11. 8 HEINICH Nathalie. Être écrivain, création et identité. Paris : La Découverte, 2000.

9 LIPOVETSKY Gilles. «Le règne de l'hyperculture: cosmopolitisme et civilisation occidentale " dans LIPOVETSKY, Gilles et Hervé JUVIN. L'Occident mondialisé. Controverse sur la culture planétaire. Paris : Éditions Grasset \& Fasquelle, 2010, p. 11-121. 
Après avoir répertorié les articles parus dans des revues spécialisées en arts visuels pendant ou immédiatement après les deux expositions, soit entre 2012 et 2015, nous avons procédé à une analyse comparative, quantitative et qualitative des articles publiés en français et en anglais. Il s'agissait, d'une part, d'évaluer la pertinence de l'hypothèse selon laquelle la réception critique francophone accuserait un déficit. Au-delà des nuances qui s'imposent d'un auteur à l'autre, nous avons, d'autre part, dressé un portrait général des contenus critiques à partir de leurs traits communs et de leurs angles morts. Nous convenons d'emblée qu'il n'était pas possible pour les auteurs d'aborder de façon exhaustive les enjeux, la diversité des thèmes et des cadres conceptuels que soulevaient ces expositions. Il va sans dire que ces comparaisons n'obéissent pas à une logique dénonciatrice ou de condamnation. Nous avons surtout cherché à dégager des points de convergence ou des recoupements qui témoignent de préoccupations communes par une majorité d'auteurs.

\section{Rareté des écrits}

Le premier constat général se dégageant de l'analyse quantitative est la rareté des textes et une prédominance d'articles portant sur Sakahàn en comparaison du nombre de commentaires critiques se rapportant à Beat Nation. Cette disparité entre le nombre d'articles consacrés à l'une et l'autre exposition n'est pas surprenante; elle paraît proportion-

10 SABET Aseman. "Catégorisations heuristiques: l'art contemporain autochtone au Québec ». Esse arts + opinions. № 81, 2014, p. 108-117.

11 VAN HOOF Marine, John K. GRANDE, Sylvia RUSSO, André SELEANU, Dorota KOZINSKA. «Dossier Art indigène $\%$. Vie des arts. $\mathrm{N}^{\circ}$ 232, 2013, p. 24-37. 12 REDGRAVE Veronica. "Beat Nation. Art, Hip-hop and Aboriginal Culture ". No 233, hiver 2013-2014, p. 60-61. PAQUIN Julio, « Beat Nation. Un manifeste artistique ». Vie des arts vol. LVIII, ${ }^{\circ} 233$, hiver 2013-2014. Paquin y présente l'exposition comme un commentaire politique, tandis que Veronica Redgrave tente de mettre en valeur une logique de mixité qui, selon elle, serait mise de l'avant par les créateurs regroupés dans cette exposition.

13 Bryne McLaughlin y rédige deux articles sur Sakahàn: McLAUGHLIN Bryne. " Curator Q\&A: How Indigenous Art Took Centre Stage in Sakahàn National Gallery of Canada, nelle à l'envergure respective des deux événements. Sakahàn réunissait près de trois fois plus d'artistes que Beat Nation et son caractère international était plus fortement marqué. Sans grand étonnement, nous avons également constaté un nombre plus important de commentaires en anglais qu'en français. Cet écart reflète à la fois le poids démographique des communautés linguistiques francophone et anglophone propre au contexte canadien et la répartition géographique des nations autochtones vivant au Québec, en Ontario et dans les provinces de l'Ouest. D'ailleurs, en introduction de son article pour la revue Esse, Aseman Sabet rappelle à juste titre les statistiques probantes à ce sujet ${ }^{10}$. Il n'en demeure pas moins que les textes parus en français ne sont pas en reste. Sakahàn inspire, par exemple, aux rédacteurs de Vie des arts la réalisation d'un dossier « indigène » bilingue publié en $2013^{11}$. L'année suivante, la même revue consacre à Beat Nation une attention comparable ${ }^{12}$. Du côté anglophone, Canadian Art est la revue qui a publié le plus grand nombre d'articles portant sur les deux expositions ${ }^{13}$. Ces efforts rejoignent les mandats respectifs de Vie des arts et de Canadian Art qui visent la couverture de l'actualité en art contemporain au Canada. Par ailleurs, fait important, les auteurs francophones sont tous allochtones, tandis que la majorité des auteurs anglophones est autochtone.

Les autres revues canadiennes spécialisées en arts visuels et dont la vocation se distingue par leur engagement idéologique ou par leurs

Ottawa May 17 to September 2, 2013 ». Canadian Art, 23 mai 2013. http://canadianart.ca/features/sakahan-national-gallery-of-canada/ McLAUGHLIN Bryne. «Sakahàn: International Indigenous Art. National Gallery of Canada, Ottawa May 17 to September 2, 2013 ». Canadian Art. Automne 2013. http://canadianart.ca/features/sakahan-national-gallery-of-canada/ Deux autres textes sont également consacrés à Beat Nation, l'un signé Tanya Harnett (membre de la nation Carry-The-Kettle, Saskatchewan) dans lequel l'auteure propose une analyse critique de la version présentée à Vancouver et l'autre de Leah Sandals qui publie une entrevue intéressante avec la commissaire Tania Willard. HARNETT, Tanya. «Beat Nation ». Canadian Art. vol. 29, n 3, 2012, p. 180. LEAH Sandals. "Q\&A. Tania Willard on Life Beyond Beat Nation ". Canadian Art, 28 juin 2013. http://canadianart. ca/features/tania-willard-beat-nation/ 
contenus thématiques sont celles qui ont réservé à ces expositions les analyses les plus approfondies, rédigées par des commentateurs autochtones anglophones ${ }^{14}$ et allochtones francophones. Du côté francophone, les revues Esse arts + opinions et Etc consacrent chacune des articles qui portent en réalité sur des sujets plus étendus que ces seules expositions, mais qui incluent néanmoins des analyses percutantes de Sakahàn ou de Beat Nation. Esse publie un texte dont l'objectif est de dresser un bilan des grands enjeux de l'art autochtone contemporain, examinant pour ce faire un éventail large d'événements parmi lesquels on mentionne Sakahàn et Beat Nation ${ }^{15}$. Etc accorde une certaine visibilité à Beat Nation dans un article qui porte également sur le travail de Nadia Myre ${ }^{16}$.

Sakahàn a, de plus, fait l'objet d'un article par l'historien de l'art d'origine australienne Henry Skerritt, paru en anglais dans The Journal of Curatorial Studies ${ }^{17}$. Pour la période étudiée, nous n'avons dénombré aucun article en français soumis à un processus d'évaluation par les pairs. Au moment de rédiger cet article, aucun texte portant sur l'exposition Beat Nation n'était paru dans une revue scientifique. Il importe toutefois de signaler la recension du catalogue de Sakahàn publiée dans RACAR, Revue d'art

14 HILL Richard William. "A column of frank reviews of recent exhibitions of Indigenous art. Sakahàn: International Indigenous Art National Gallery of Canada 17 May-2 September 2013 ». Fuse Magazine. Vol. 36, nº 4, 2013 , p. 40-42. NANIBUSH Wanda. «Sakahàn: International Indigenous Art ». C: International Contemporary Art. $\mathrm{N}^{\circ} 119$, 2013, p. 54-55. DAVIDGE Michael. « Sakahàn, International Indigenous Art ". Border Crossings, vol. 32, n 4, 2013, p. 83. Sakahàn fait l'objet d'une très brève mention promotionnelle de l'exposition, la seule dans une revue américaine, VAN DYKE Kristina. "Sakahan: international indigenous art ". Art forum International. Vol. 51, n 9, 2013, p. 163. Seule Kristina Van Dyke n'est pas autochtone. Elle rédige sur Sakahàn un court encadré promotionnel.

15 SABET Aseman. "Catégorisations heuristiques: l'art contemporain autochtone au Québec ". Esse arts + opinions. No 81, 2014, p. 108-117.

16 BOUCHARD Anne-Marie. " Nadia Myre, The Scar Project et Beat Nation ». ETC, $\mathrm{n}^{\circ}$ 101, 2014, p. 40-43. Notons que Nadia Myre ne faisait pas partie de l'exposition Beat Nation. 17 SKERRITT Henry. "Sakahàn Indigenous Art ». Journal of Curatorial Studies. Vol. 3, nos 2-3, 2014, p. 395-399. Henry Skerritt est historien de l'art et conservateur, Mellon Curator of Indigenous Arts of Australia, de la collection Kluge-Ruhe Aboriginal Art Collection de l'Université de Virginie.
canadienne/Canadian Art Review ${ }^{18}$ ainsi que les articles respectifs de Laurent Jérôme et Jean-Philippe Uzel ${ }^{19}$ qui se penchent sur d'autres sujets, mais qui soulignent tous deux l'importance de ces expositions.

\section{Regards croisés et angles morts}

Les auteurs reconnaissent tous la nécessité d'une ouverture du cadre d'analyse de la production autochtone pour y inclure d'autres voix que celles de l'histoire de l'art canonique. Prenant souvent appui sur les propos de Christine Lalonde, plusieurs auteurs avancent l'idée d'une " citoyenneté culturelle autochtone $^{20}$ " qui va au-delà des limites géographiques nationales et de la cartographie arbitraire tracées par l'histoire coloniale. On souligne parfois la sous-représentation de certains groupes. Dans sa recension du catalogue de Sakahàn, Véronique Gagnon déplore le fait que plusieurs territoires majeurs manquent à l'appel - les peuples autochtones de Russie, de Chine et d'Afrique notamment ${ }^{21}$. Dans la section dévolue à Beat Nation de l'article rédigé pour la revue Etc, Anne-Marie Bouchard mentionne la sous-représentation des artistes provenant du Québec. Il est vrai que la version montréalaise

18 GAGNON Véronique. "Recension. Greg A. Hill, Candice Hopkins et Christine Lalonde (dir.), Sakahàn : Art indigène international, catalogue d'exposition. Ottawa: Musée des beaux-arts du Canada, 2013 ". RACAR Revue d'art canadienne/Canadian Art Review. vol. 39, $\mathrm{n}^{\circ}$ 2, 2014, p. 121-125.

19 JÉROME Laurent. "Présentation: vues de l'autre, voix de l'objet dans les musées ". Anthropologie et Sociétés. Vol. 38, $\mathrm{n}^{\circ}$ 3, 2014, p. 9-15. UZEL Jean-Philippe. "L'autochtonie dans l'art actuel québécois. Une question partagée ". Globe: revue internationale d'études québécoises, vol. 17, $\mathrm{n}^{\circ} 1,2014$, p. 33-57.

20 LALONDE Christine. "Introduction: au carrefour de l'indigénéité, de la mondialisation et de l'art contemporain " dans HILL, Greg A., Candice HOPKINS, Christine LALONDE et al. Sakahàn Art indigène international. Ottawa : Musée des beaux-arts du Canada, 2013, p. 13-20. 21 GAGNON Véronique. "Recension. Greg A. Hill, Candice Hopkins et Christine Lalonde (dir.), Sakahàn: Art indigène international, catalogue d'exposition. Ottawa: Musée des beaux-arts du Canada, 2013 ». RACAR Revue d'art canadienne/Canadian Art Review. vol. 39, n 2, 2014, p. 122. 
de Beat Nation n'incluait aucun artiste né au Québec, bien qu'y figuraient Raymond Boisjoly (d'ascendances québécoise et haida) et Sonny Assu, artiste laich-kwil-tach très actif sur la scène montréalaise.

Les auteurs ont été nombreux à souligner ce qu'ils ont perçu, de prime abord, comme l'absence d'un fil conducteur qui aurait pu guider la lecture de Sakahàn. Afin de pallier l'effet déstabilisateur souvent ressenti, certains auteurs tentent de repérer des thèmes qui émergeraient de l'agencement des œuvres en salles d'exposition. C'est le cas notamment de Marine Van Hoof pour Vie des arts. Cette auteure organise son texte en reprenant les quelques lignes directrices esquissées par les panneaux didactiques, notamment la "valorisation du fait main » ou les « rapports entre le spirituel et le quotidien ". À son avis, ce serait toutefois la " conception animiste omniprésente qui serait le fil conducteur ${ }^{22}$ " de l'exposition. Sans douter des intentions bienveillantes de cette remarque, il faut admettre qu'en l'absence d'une mise en contexte théorique, la notion d'animisme peut mener le lecteur à une interprétation essentialiste que l'auteure prend pourtant bien soin de décrier. D'autres commentateurs échappent à toute pulsion de catégorisation et voient plutôt l'apparente absence de trame narrative de Sakahàn comme une stratégie pouvant engendrer de fécondes remises en question de nos schèmes de pensée. À ce sujet, Richard William Hill note:

\section{[...] many other (galleries) remained a mys- tery to me. This may be because some of the juxtapositions were more visually poetic than explicitly thematic and I was searching in vain, or perhaps it is just that I have trouble}

processing a lot of information at once and never made all the connections. I suspect the curators took this more subtle approach because this is an exercise in getting to know one another and they wanted to leave things open $^{23}$.

Voulant explorer la sensibilité hip-hop comme forme d'activisme, les œuvres présentées dans Beat Nation étaient, quant à elles, clairement regroupées par sous-thèmes : beat (pulsion), stage (scène), street (rue) et tag (marqueur). La majorité des auteurs s'avère sensible à la polysensorialité des projets artistiques, au métissage des espaces, tel que préconisé par les commissaires ${ }^{24}$, au brouillage des repères spatiaux et aux identités troubles qui émergent de ces environnements. On souligne, par exemple, l'érosion des frontières disciplinaires et la fluidité des contenus de la vidéo de Nicholas Galanin que Robin Laurence, pour Border Crossings, rapproche de la figure du trickster ${ }^{25}$. Du côté francophone, Anne-Marie Bouchard abonde dans le même sens, tout en faisant ressortir, du même coup, les paradoxes du hiphop comme thème. Après avoir souligné les apparentes contradictions entre la culture " misogyne " du hip-hop et le rôle central qu'occupent les femmes dans les cultures autochtones, elle se demande si le langage formel et symbolique du hip-hop " fragilise la transmission des cultures autochtones " ou, au contraire, s'il contribue à les " médiatiser plus efficacement ». À son avis, cette question s'inscrit en filigrane de toute l'exposition, puisque "l'adoption de références au hip-hop se double d'une adoption de moyens d'expression stratégiquement choisis parmi les recettes les plus efficientes des arts médiatiques ${ }^{26}$."
22 VAN HOOF Marine. "Sakahàn. Dialogue du présent avec le passé ". Vie des arts. vol. 232, 2013, p. 27. 23 "Certaines des juxtapositions étaient visuellement plus poétiques que explicitement thématiques et je cherchais en vain, peut-être ai-je simplement de la difficulté à intégrer un grand nombre d'informations à la fois et à faire tous les liens. Je soupçonne que les commissaires aient adopté cette approche plus subtile, parce qu'il s'agit d'un exercice qui vise à mieux se connaître les uns et les autres. " (Traduction libre). HILL, Richard William. "A column of frank reviews of recent exhibitions of Indigenous art. Sakahàn: International Indigenous Art National Gallery of Canada, 17 May-2 September 2013 ». Fuse Magazine. Vol. 36, n 4, 2013, p. 42. 24 HARNETT Tanya. "Beat Nation ". Canadian Art, Vol. 29, n 3, 2012, p. 180.

25 LAURENCE Robin. «Beat Nation ». Border Crossings, Vol. 31, n 3, 2012, p. 120.

26 BOUCHARD Anne-Marie. « Nadia Myre, The Scar Project et Beat Nation ", ETC, $\mathrm{n}^{\circ}$ 101, 2014, p. 43. 


\section{Controverses autour de la notion d'« indigénéité »}

La bannière " raciale " sous laquelle sont présentées les deux expositions, particulièrement Sakahàn, provoque un malaise tangible. À ce sujet, les auteurs se montrent attentifs aux intentions des commissaires de Sakahàn et à leur usage stratégique de la notion d'" essentialisme ". Rappelons que les commissaires et les auteurs du catalogue ont voulu promouvoir une vision renouvelée de l'« indigénéité ». Pour ce faire, Greg Hill et Jolene Rickard mettent de l'avant l'idée empruntée à Gayatri Chakravorty Spivak d'un usage conscient, concerté, temporaire, politique et stratégique de l'essentialisme ${ }^{27}$. Envisagé de la sorte, « l'essentialisme stratégique " renverrait davantage à une puissance d'agir qu'à une définition identitaire. Pour Fuse, Richard William Hill (Cri) présente une analyse approfondie et percutante des notions d'indigénéité et de l'essentialisme stratégique. Se méfiant des postures ou des interprétations qui pourraient mener à l'enfermement identitaire et à de vieux réflexes essentialistes ou fétichistes hérités de la modernité, il soutient:

"These terms worry me because they seem to lose track of the distinction between institutional arrangements, which are inevitably blunt instruments, and the more subtle and promiscuous movements of culture ${ }^{28}$ ". Wanda Nanibush (Anishinaabe-kwe) rappelle, quant à elle, la nécessité de ne pas perdre de vue l'impact d'un tel horizon élargi de l'indigénéité sur les communautés locales d'ici: «Without grounding the global in a local, the political use of Indigenous, as Jolene Rickard points out, gets lost ${ }^{29}$ ". Enfin, dans The Journal of Curatorial Studies, l'auteur australien Henry Skerritt loue la capacité de Sakahàn à générer des réponses personnelles au traumatisme colonial et à créer des « histoires parallèles ». Toutefois, l'absence de " créateurs-

27 HILL Greg. "Postface. Sakahàn, 25 ans après " dans HILL Greg, Candice HOPKINS, Christine LALONDE, Sakahàn art indigène international, Ottawa: Musée des beaux-arts du Canada, 2013, p. 135-140. RICKARD, Jolene. " L'émergence de l'art indigène international " dans HILL Greg, Candice HOPKINS, Christine LALONDE, Sakahàn art indigène international, Ottawa: Musée des beaux-arts du Canada, 2013, p. 53-60. artisans " renforcerait, selon lui, les prémisses euroaméricaines qui définissent l'art contemporain et relègueraient l'art autochtone à " un statut inférieur ${ }^{30} "$.

\section{Hors mur, engagement citoyen et sororité}

Simultanément aux œuvres montrées en salle, Sakahàn se prolongeait dans plusieurs autres lieux de diffusion: dans l'espace public de la ville d'Ottawa, à Winnipeg ainsi qu'à Wellington en Nouvelle-Zélande. Quelques auteurs se montrent attentifs à cette dimension. Par exemple, constatant les difficultés pratiques à « décoloniser » le musée, Henry Skerritt souligne l'intérêt et la force de la murale extérieure, mais adjacente au musée, Salmon cycle The Spirit Whithin (2013), de Corey Bulpitt et Larissa Healey ${ }^{31}$. Véronique Gagnon mentionne le fait que Sakahàn s'étendait à l'extérieur des murs du Musée des beaux-arts du Canada. Toutefois, la nature de son texte (une recension de catalogue) ne lui permet pas d'approfondir une analyse des enjeux pragmatiques ou conceptuels se rattachant à cette composante hors murs, collective et structurante. Michael Davidge (pour Border Crossings) est l'auteur le plus attentif aux espaces hors murs de l'exposition Sakahàn qu'il décrit assez longuement. Il note, entre autres choses, l'insertion des affiches de l'artiste groenlandaise Julie Edel Hardenberg, tirées de sa série Sapiitsut/ Heroes (2011). Davidge se penche aussi sur la stratégie de résistance que proposent certains accrochages furtifs qui s'inscrivent en porte-à-faux à l'architecture du musée et du site comme symboles forts de la nation canadienne. C'est le cas notamment de la bannière de Nadia Myre (For those who cannot speak: the land, the water, the animals and future generations, 2013) qui fait littéralement corps avec l'architecture du

28 HILL Richard William, op. cit., p. 42.

29 NANIBUSH Wanda. "Sakahàn: International Indigenous Art ". C: International Contemporary Art. n 119 , 2013, p. 55.

30 SKERRITT Henry, op. cit., p. 398.

31 Ibidem. 
musée et qui exprime la solidarité de l'artiste envers les kokoms (les grands-mères algonquines). Le titre de l'œuvre fait explicitement référence à la déclaration que ces dernières ont lue, le 11 janvier 2013, sur la colline du Parlement en réaction au projet de loi omnibus (Loi C45) de l'administration conservatrice sous la gouverne de Stephen Harper qui touchait notamment les droits d'accès à l'eau et au territoire. Au moment de l'exposition, le cartel accompagnant l'œuvre de Nadia Myre comportait une brève note dissociant le musée des propos de l'artiste, clairement solidaires des déclarations des femmes autochtones visant à dénoncer les politiques gouvernementales qui confinent au mutisme " la terre, les eaux, les animaux et les générations futures ". C’est dire, que ironiquement, le cartel d'origine faisait implicitement écho aux attitudes répressives dénoncées par les manifestantes et par le titre du projet de Nadia Myre. Se délestant de la charge contestataire du travail de Nadia Myre, la note d'origine, reconduisait en effet des procédures et des protocoles institutionnels qui contribuent traditionnellement à une forme de déresponsabilisation. Or, est-il besoin de rappeler qu'il s'agit ici d'un musée d'État. Du coup, paradoxalement, plutôt que d'affaiblir la charge politique de l'œuvre de Myre, le cartel d'origine la renforçait car d'une certaine façon, elle constituait une démonstration probante, quoique non intentionnelle, des attitudes d'indifférence devant le mutisme qu'évoquent tant la proposition de l'artiste que les déclarations des kokoms. Curieusement aucun des textes recensés n’a dénoté cette antiphrase.

D'autres projets artistiques structurants et collectifs menés par des artistes en collaboration avec des femmes de leurs communautés - (Récits de couvertures (2013) de Marie Watt (Sénéca), Sur les ailes du rêve (2002) de Yuma Taru (Atayal), ou encore Femmes brodant près du lac Atitlan (2012) de Teresa Margolles
(Mexicaine), par exemple - ont été mis en œuvre plus spécifiquement dans l'espace public, citoyen et local. Ces processus collaboratifs reçoivent relativement peu d'attention de la part de la critique. Pourtant, leurs ancrages dans l'oralité au sein de communautés locales de femmes revêtent un réel potentiel de changements. Seule Wanda Nanibush insiste, comme nous l'avons mentionné plus haut, sur l'importance d'un ancrage « local » dans un monde globalisé sans toutefois rattacher précisément ce commentaire aux processus collaboratifs et à la sororité que sous-tendent certains projets artistiques.

Enfin, les commentateurs ne s'attardent pas aux enjeux théoriques et politiques que soulèvent les remaniements et la structure dynamique, voire intermédiale (du blogue vers la galerie autogérée et le musée) du " projet " Beat Nation $^{32}$. Rappelons que dans sa genèse, le projet initial conçu par Tania Willard et Skeena Reece (membre fondateur du Native Youth Artists Collective) en collaboration avec Glenn Aleen, directeur du centre d'artistes autogéré Grunt Gallery a été réalisé sur le Web en 2008 (www.beatnation.org). L'exposition en ligne mettait en valeur des créateurs multidisciplinaires (musiciens, artistes visuels, écrivains) inspirés de la sensibilité hip-hop, du breakdance, des graffitis, du disc-jockey et des activités de maîtres de cérémonie issus des territoires Salish non cédés de la côte ouest. Le projet Web a été réalisé grâce au Fonds de la passerelle octroyé par le ministère du Patrimoine canadien pour le soutien à des initiatives qui font partie de la stratégie de Culture canadienne en ligne. Cette première version de Beat Nation, qui comprenait un blogue interactif, a ensuite été remaniée afin d'être présentée sous forme d'exposition relativement modeste à Ottawa, à la SAW Gallery (du 21 avril au 26 juin 2009). Par la suite, Kathleen Ritter, conservatrice adjointe associée à la Vancouver Art Gallery, a 
invité Tania Willard à coproduire une version plus ambitieuse de l'exposition. Notons qu'à ce moment, la co-initiatrice du projet Skeena Reece ne faisait plus partie de l'équipe commissariale. La Vancouver Art Gallery a donc présenté Beat Nation du 25 février au 3 juin 2012 et elle a organisé du même coup la mise en circulation de cette version. L'exposition a ensuite voyagé dans plusieurs villes canadiennes ${ }^{33}$. C'est dire que selon des modalités et des stratégies différentes, l'exposition Beat Nation comportait, elle aussi, une forte dimension hors murs qui échappe majoritairement au regard de la critique.

Finalement, force est de constater qu'en général, on reste peu bavard sur la centralité de l'engagement et la responsabilisation des citoyens desquels émergent plusieurs projets artistiques montrés dans Sakahàn et Beat Nation. Or, l'ancrage communautaire, souvent féminin, les interventions dans des territoires virtuels et dans des interstices urbains ainsi que les dimensions immatérielles des projets collaboratifs constituent autant de facteurs qui contribuent justement à un processus d' « indigénisation " des savoirs.

\section{Épilogue, pour ne pas conclure}

Quelques constats se dégagent de ces préoccupations communes et de ces angles morts. L'exercice comparatif montre, d'une part, que les auteurs ont unanimement apprécié la qualité des œuvres présentées et qu'ils ont accueilli positivement la tenue de ces deux expositions majeures dans le paysage de l'art contemporain au Canada. On reconnait, d'autre part, que ces expositions étaient des occasions concrètes de valoriser l'internationalisation du réseau de

33 De Vancouver, l'exposition a voyagé à: Toronto, Power Plant Contemporary Art Gallery (du 15 décembre 2012 au 5 mai 2013), Kamloops, Art Gallery (du 29 juin au 7 septembre 2013), Montréal, Musée d'art contemporain (du 10 octobre 2013 au 2 janvier 2014), Halifax, Dalhousie Art Gallery and St. Mary's University Gallery (du 22 mars au 18 mai 2014), Régina, Makenzie Art Gallery (du 5 juillet au 7 septembre 2014).

34 HILL Richard William, op. cit. p. 42. En fait, cette voie collaborative était présente dans Sakahàn. La bannière l'art autochtone contemporain. Les auteurs se sont majoritairement intéressés à la notion d'" indigéniété stratégique " qui a suscité chez nombre d'entre eux des réserves quant aux dérives essentialistes auxquelles elle peut mener. Les commentateurs autochtones anglophones ont été plus nombreux à soulever de façon approfondie cette épineuse question et à proposer des solutions de rechange. Afin d'éviter tout écueil essentialiste, Richard William Hill conclut son essai en conviant le lecteur à aborder «l'indigénéité » non pas comme identité, mais comme thème: "Another possibility would be to think of "Indigenous" as a theme rather than the identity of the participants, and open it up further to potential non-indigenous allies whom we would like to bring into the conversation $^{34} »$. Envisager l'indigénéité comme thème, ou comme " récit de soi " pour reprendre l'expression de Judith Butler, reviendrait sans doute à mettre l'accent sur le caractère narratif et relationnel de l'identité ainsi que sur la capacité et la responsabilité du « je » à aborder les normes sociales de façons « vivantes et réflexives ${ }^{35}$ ".

Les critères d'appréciation sur lesquels se fondent de nombreux commentaires sont issus d'un discours critique spécialisé fortement attaché à une sociologie de l'art assez classique. On se demande, par exemple, qui expose, on cherche à identifier les grands thèmes des expositions et à saisir l'intentionnalité des commissaires. On s'intéresse donc principalement au contexte institutionnel de médiation et de diffusion de l'art, et plus rarement au contexte des interactions sociales et locales qui précèdent à la production et à la réalisation des œuvres et à ses liens avec le musée. Aussi importants soientils, ces critères d'appréciation servent parfois à satisfaire «l'exigence de cohérence qu'enferme

intitulée Terre et ciel était la seule œuvre réalisée conjointement par une artiste autochtone Shuvinai Ashoona (Inuit, Cape Dorset) et un artiste allochtone, John Noestheden (allochtone, Canadien et Néerlandais). Nous remercions le commissaire Greg Hill d'avoir porté à notre attention cette dimension. Entrevue téléphonique de l'auteure avec G. Hill, le 22 mai 2015.

35 BUTLER Judith, Le récit de soi. Paris: Presses universitaires de France, (2007) 2010, p. 10. 
l'idée de l'art ${ }^{36}$ ". La cohérence de cette exigence est, comme l'explique Becker de " continuer à apporter une caution logique à ce que le public perçoit comme des œuvres d'art importantes, et maintenir ainsi une relation cohérente entre ce qui est déjà avalisé de longue date et ce qui est proposé maintenant ${ }^{37}$ ".

Bien que les auteurs se penchent respectivement sur certains des enjeux politiques que soulèvent ces expositions, il reste que le caractère immatériel des échanges citoyens sur lequel repose le processus créatif de plusieurs œuvres, la sororité sous-jacente aux projets de certaines créatrices ainsi que l'éclatement des lieux d'intervention, sont plus rarement abordés. Or, ces dimensions constituent autant de défis que posent les arts autochtones contemporains à la muséologie et à la critique d'art.

En ce qui concerne l'hypothèse du déficit francophone, nous avons souligné le fait que le nombre plus faible d'articles en français reflète le poids des communautés linguistiques canadiennes et la répartition géographique des nations autochtones habitant les territoires qui forment le Canada. Cette représentativité démographique vient nuancer les préjugés selon lesquels la communauté francophone au Québec manque de sensibilité au regard des réalités autochtones. Il n'en demeure pas moins, et cela malgré l'effervescence des projets artistiques autochtones au Québec et le dynamisme des initiatives visant leur mise en valeur, que les artistes autochtones qui parlent français peinent à être connus et reconnus, occupant en quelque sorte une zone d'intersectionnalité discriminatoire $^{38}$ au regard de la langue et de la culture. Plusieurs intervenants ont, avec raison, décrié leur quasi « invisibilité ${ }^{39}$ dans le champ dominant de l'art contemporain.
En dépit de la qualité des articles recensés et compte tenu de l'importance de ces expositions, Sakahàn et Beat Nation ont généré, somme toute, peu d'analyses critiques tant dans les revues spécialisées anglophones que francophones. Ce déséquilibre entre la vitalité de la scène artistique autochtone et sa réception critique dans les revues spécialisées semble être généralisé et dépasser les seules tensions linguistiques propres au contexte canadien. Dans un article de 2012 portant sur Alex Janvier, Richard William Hill, qui écrit pour le magazine anglophone Fuse, se plaint d'une telle inadéquation: "I proposed this column to FUSE because I was concerned about the poverty of critical response to recent exhibitions of contemporary Indigenous art ${ }^{40}$ ". Compte tenu des limites de cet essai, il est impossible de mesurer l'étendue de cette carence ou d'en évaluer comparativement les impacts selon les milieux linguistiques francophones ou anglophones. Toutefois, on peut penser que les conditions souvent précaires de production dans lesquelles travaillent les diffuseurs en art contemporain constituent un des facteurs expliquant la tendance actuelle de plusieurs revues spécialisées en arts visuels à concevoir des numéros thématiques, une stratégie qui laisse moins de place aux recensions critiques approfondies portant sur un événement ponctuel, autochtone ou non. Comme le remarque Sylvette Babin, éditrice de la revue Esse, le sous-financement et la compétition féroce auxquels font face les revues spécialisées en arts visuels au Québec ont contribué au développement de nouvelles stratégies, parmi lesquelles figure le numéro à thèmes ${ }^{41}$. Contrairement aux revues constituées d'articles libres, ce choix éditorial présente l'avantage de créer une spécificité à la revue, une niche qui s'avère compétitive. Cependant, cette stratégie éditoriale crée une certaine

examen de la connaissance et de la documentation, 2011, Ottawa: Conseil des arts du Canada. TRÉPANIER, France et Chris CREIGHTON-KELLY. "La langue de l'autre (The Language of the Other)". Revue d'art canadienne/ Canadian Art Review, vol. 41, n 1, 2016, p. 37-41. 40 HILL, Richard William. "Closed Readings. Alex Janvier ". Fuse, vol. 6, n 2, 2012, p. 44.

41 Conversation téléphonique de l'auteure avec Sylvette Babin, le 25 mai 2015.

38 Nous faisons ici référence au concept développé par Kimberley Crenshaw dans son article aujourd'hui célèbre, "Mapping the Margins: Intersectionality, Identity, Politics, and Violence against Women of Color ", Stanford Law Review vol. 43, nº 6, 1991, p. 1241-1299. 
pénurie d'analyses critiques portant sur l'actualité des expositions autochtones et allochtones. Dans ce contexte, il n'est pas surprenant que du côté anglophone ce soit Canadian Art et Vie des arts, du côté francophone, qui aient consacré à Sakahàn et à Beat Nation les dossiers les plus longs. Contrairement à d'autres diffuseurs qui réalisent des dossiers thématiques de façon systématique, Vie des arts, tout comme Canadian Art, a choisi de répondre à sa mission en " rendant compte de la pluralité et de la diversité de la production de nos artistes ${ }^{42}$ " et en se penchant sur un large éventail d'événements différents dont les enjeux et les thèmes sont hétéroclites.

Finalement, plusieurs auteurs reconnaissent la nécessité d'un recadrage conceptuel, théorique, voire épistémologique, allant dans le sens d'une réforme de nos systèmes de pensée, de nos espaces d'exposition et du discours critique luimême. À des degrés divers, cette mobilisation est palpable chez tous les auteurs. Les critiques insistent sur le besoin d'ouverture des cadres processus "d'indigénisation » des structures institutionnelles muséales. À ce sujet, Aseman Sabet conclut: « Si nous changeons nos habitudes méthodologiques, modifions nos tendances catégorielles rigides et ouvrons la recherche à une approche plus empathique et dialogique, plusieurs réponses viendront ${ }^{43}$ ". Bien que l'échantillonnage étudié dans cette étude soit relativement restreint, sa diversité (textes en français, en anglais, auteurs autochtones et allochtones) le rend représentatif des différentes postures de la critique d'art au Canada. Il n'est donc pas exagéré de voir dans les questionnements épistémologiques qui nourrissent les commentaires des auteurs, une réelle prise de conscience de la nécessité de redéfinir les approches muséologiques et critiques, soit quelque 20 ans après les recomman-

42 http://viedesarts.com/A+propos (consulté le $1^{\text {er }}$ avril 2015)

43 SABET, Aseman, op. cit., p. 117.

44 Rappelons-nous qu'au sens de la loi, le Canada est, encore aujourd'hui, un système colonial et non postcolonial.

dations formulées en 1992 par le Groupe de travail sur les musées et les Premières Nations et en 1996, par la Commission royale sur les peuples autochtones. Il faut toutefois se rendre à l'évidence que les approches conceptuelles capables de prendre en compte de tels déplacements théoriques dans le contexte géopolitique colonial $^{44} \mathrm{du}$ Canada sont en construction.

Nous ne faisons que commencer à récolter les fruits de ces chantiers d'études, des méthodes autochtones et des paramètres de recherche renouvelés. Les programmes universitaires d'études autochtones sont un phénomène relativement récent au Canada anglais et encore plus nouveau dans les milieux universitaires francophones où les cadres organisationnels sont encore extrêmement fragmentaires. Conjugué à la rareté des professeurs-chercheurs autochtones et à l'état parcellaire des structures organisationnelles, le sous-développement de cadres d'enseignement postsecondaire adaptés aux candidats autochtones ${ }^{45}$ nuit à la construction d'une muséologie et d'un discours critique véritablement capable de prendre en compte les défis que posent les arts autochtones au champ de l'art. Ces faiblesses structurales et organisationnelles contribuent assurément à expliquer la persistance de certains stéréotypes langagiers et des angles morts constatés dans notre étude. Elles expliquent également, en partie, l'absence de commentateurs francophones autochtones.

Nous avons entamé cette analyse et ce dépouillement de la réception critique de Sakahàn et Beat Nation en nous intéressant aux diffuseurs spécialisés parce qu'ils jouent un rôle avéré dans le processus de reconnaissance de l'art contemporain. Nous voulons pour conclure signaler les limites de notre propre démarche. Afin de mieux saisir le potentiel performatif du discours critique, il faudrait sans doute tenir compte de sa "multiplicité des moments ",

45 À ce sujet, on peut consulter LEFEVRE-RADELLI, Léa et Laurent JÉRÔME, Expériences, politiques et pratiques d'intégration des étudiant.es autochtones à l'université: le cas de l'UQAM, Montréal: Cercle des Premières Nations et Service aux collectivités de l'UQAM, 2017. 
pour paraphraser Becker. Cela permettrait

d'explorer les autres territoires qu'il occupe tout

en l'examinant dans ses différents temps et

modes de transmission. L'examen conjoint des

textes et de commentaires parus dans des

revues spécialisées et dans les médias alterna-

tifs (forums en ligne, blogues, réseaux sociaux ou télévision communautaire, par exemple) permettrait vraisemblablement d'aller à l'encontre des critères d'appréciation convenus, des hiérarchies des formes du discours sur l'art et d'entamer un processus critique du discours critique lui-même. 


\begin{abstract}
This article offers a qualitative and quantitive analysis of the critical reception of two exhibitions, Sakahàn:International Indigenous Art (National Gallery of Canada, Ottawa 2013) and Beat Nation: Art, HipHop and Aboriginial Culture (organised and circulated by the Vancouver Art Gallery, 2013-2014). The study treats articles which appeared between 2012 and 2015 in English and French visual-arts publications. The comparative analysis intends to highlight general trends, in order to identify challenges that contemporary Indigenous arts pose for art criticism. A review of the texts shows that all commentators, whether francophone or anglophone, indigenous or non-Indigenous, have welcomed these two exhibitions warmly. The discrepancy between the number of essays in French and those in English reflects the demographic weight of these two linguistic communities and the geographic distribution of First Nations in Canada. This will qualify, without denying, the hypothesis of Quebec's tardiness on the indigenous question. The authors largely recognize the necessity of initiating indigenization of the museum and emphasize the movement to internationalize contemporary indigenous art. Yet many commentators, particulary Indigenous people, dispute the efficacity of the concept of "strategic essentialism" put forward by the commissioners of the Sakahàn catalog. Despite both a real interest in these two major exhibitions and the quality of the commentary, in the end, for events of such a scale few texts have been published on the subject. The criteria for appreciation rooted in the institutional sociology of art endeavour to fully take into account the challenges posed by certain central aspects of the approach of several Indigenous creators, such as the intangible dimensions of their civic engagement, the dissolution of particular outside venues and the sisterhood of certain projects.
\end{abstract}

